\title{
ESTUDO DA INTERAÇÃO GENÓTIPO X AMBIENTE EM PROGÊNIES DE Pinus taeda POR MEIO DA ANÁLISE DE PARÂMETROS GENÉTICOS
}

\author{
Diego Tyszka Martinez ${ }^{1}$, Marcos Deon Vilela de Resende ${ }^{2}$, Reginaldo Brito da Costa ${ }^{1}$, \\ Antonio Rioyei Higa ${ }^{3}$, Gleison Augusto dos Santos ${ }^{4}$, Ivone Satsuki Namikawa Fier ${ }^{5}$ \\ ${ }^{1}$ Eng. Florestal, Dr., UFMT, Cuiabá, MT, Brasil - diegotyszka@ hotmail.com; reg.brito.costa@gmail.com \\ ${ }^{2}$ Eng. Agrônomo, Dr., EMBRAPA Florestas, Colombo, PR, Brasil - marcos.deon@gmail.com \\ ${ }^{3}$ Eng. Florestal, Dr., Depto. de Ciências Florestais, UFPR, Curitiba, PR, Brasil - antonio.higa@ gmail.com \\ ${ }^{4}$ Eng. Florestal, M.Sc., CMPC Celulose Riograndense, Guaíba, RS, Brasil - gadsantos@klabin.com.br \\ ${ }^{5}$ Eng. Florestal, M.Sc., Klabin, Telêmaco Borba, PR, Brasil - namikawa@ klabin.com.br
}

Recebido para publicação: 01/12/2010 - Aceito para publicação: 07/08/2012

\begin{abstract}
Resumo
O presente estudo objetivou avaliar parâmetros genéticos associados à heterogeneidade de variâncias e interação genótipos x ambientes, comparando-se os resultados obtidos pelos procedimentos Blup e Blup-Het, em testes de progênies de Pinus taeda L.. Os experimentos foram instalados sob delineamento de blocos ao acaso com 150 tratamentos, sendo três locais em Santa Catarina e dois no Paraná, com 5 e 9 blocos por local, respectivamente, 6 plantas por parcela em linhas simples. Aos 6 anos de idade, foram avaliados altura e diâmetro. No geral, os resultados mostraram que as variâncias residuais elevadas conduziram à heterogeneidade de variâncias, que, associadas às altas correlações genéticas entre os caracteres altura e diâmetro, apesar de semelhantes, indicam que o procedimento Blup-Het deve ser utilizado, pois a estimativa de parâmetros genéticos usando herdabilidade individual por progênie é mais precisa. A interação genótipos x ambientes foi baixa, em função das semelhanças entre os componentes de variância e das progênies selecionadas por critérios de adaptabilidade, estabilidade e produtividade. O programa de melhoramento genético pode ser conduzido em apenas um dos locais, tendo em vista a similaridade dos ambientes, demonstrado pelos baixos valores de coeficiente de determinação dos efeitos da interação genótipos x ambientes.

Palavras-chave: Modelos lineares mistos; ganho com seleção; herdabilidade individual; heterogeneidade de variâncias.
\end{abstract}

\begin{abstract}
Study of genotype $x$ environment interaction in progenies of Pinus taeda by analysis of genetic parameters. This study evaluated genetic parameters, heterogeneity of residual variances and genotype $\mathrm{x}$ environment interaction, comparing the results obtained by Blup and Blup-Het procedures in Pinus taeda L. progeny tests. Experiments were set in random blocks with 150 treatments. Three experiments were located in Santa Catarina state and two were set in Paraná state, Brazil, with 5 and 9 blocks per site, respectively, and six plants per plot. At 6 years old, the progenies were evaluated for height and diameter. In general, results demonstrated that high residual variance lead to variance heterogeneity. When variances are associated to high genetic correlations among height and diameter characters, they indicate that Blup-Het procedure should be used, even if they present similar results, because genetic parameters estimated based on individual heritability for progeny is more accurate. Genotype $\mathrm{x}$ environment interaction was low, given similarities among progeny variance and similarity components, selected by adaptability, stability and productivity criteria. The genetic improvement program may be conducted at one site, due to the environments similarities, as demonstrated by effects from genotype $\mathrm{x}$ environment interaction determination coefficient and genetic correlations across sites.

Keywords: Selection gain; linear mixed models; individual heritability; heterogeneity of variances.
\end{abstract}

\section{INTRODUÇÃO}

O gênero Pinus insere-se de forma significativa na economia de regiões de clima subtropical no Brasil, em especial nos estados do Paraná e Santa Catarina, ocupando, segundo dados da ASSOCIAÇÃO BRASILEIRA DE PRODUTORES DE FLORESTAS PLANTADAS (2011), uma área plantada de 
1.756.359 ha em 2010. Entre as espécies plantadas, o Pinus taeda L. tem se destacado desde sua introdução no país, quando comparado com outras espécies do mesmo gênero, devido ao seu bom crescimento e elevada qualidade da madeira, utilizada na produção de celulose de fibra longa pelo processo Kraft, na produção de madeira serrada, laminados, chapas e compensados, com ampla adaptação nas regiões de plantio.

As principais características avaliadas em programas de melhoramento de Pinus taeda são relacionadas à produtividade e à qualidade da madeira, de acordo com o objetivo da produção. Para celulose, aspectos relacionados à produtividade volumétrica e a qualidade da madeira são utilizados como critérios de seleção em alguns trabalhos de melhoramento. A avaliação das variáveis diâmetro e altura vem sendo utilizada em testes genéticos para prever a produtividade e estimar os parâmetros e ganhos com seleção. $\mathrm{O}$ desempenho dos materiais genéticos em diferentes locais permite avaliar a interação genótipos $\mathrm{x}$ ambientes e obter informações sobre adaptabilidade (DEMERITT; GARRET, 1996), além de estabilidade e produtividade. Segundo Paludzyszyn Filho et al. (2001), quando os testes de progênies são realizados em vários locais, se caracterizam o desempenho relativo das progênies e a qualidade do ambiente, revelando a interação das progênies com os diferentes locais quando há diferenciação entre os ambientes.

Para a análise e estimação dos parâmetros genéticos, atualmente tem sido utilizada a metodologia Reml/Blup (máxima verossimilhança restrita e melhor predição linear não viciada), que permite predizer os valores genéticos mesmo em casos de experimentos desbalanceados. Essa metodologia funciona bem em casos de variâncias residuais homogêneas, em que a herdabilidade média do experimento se aproxima da herdabilidade média do genótipo. Em casos com heterogeneidade de variâncias residuais dentro de tratamentos, o uso de uma herdabilidade média do experimento ocasiona subestimativa ou superestimativa na predição dos valores genéticos. Nesse caso, o uso do procedimento Blup-Het (disponível no software Selegen-Reml/Blup), que estima uma herdabilidade para cada genótipo, torna-se mais adequado (RESENDE; DUARTE, 2007).

O presente estudo objetivou avaliar os parâmetros genéticos, heterogeneidade de variâncias e interação genótipos $\mathrm{x}$ ambientes através dos procedimentos Blup e Blup-Het para caracteres de crescimento em progênies de Pinus taeda e definir a melhor estratégia para o programa de melhoramento genético.

\section{MATERIAL E MÉTODOS}

O estudo foi realizado por meio de análise genético-estatística da rede experimental de Pinus taeda da empresa Klabin S.A., composta por 150 progênies de meio-irmãos, originadas de cinco populações (Mondi I, Mondi II, Zimbábue I, Zimbábue II, Pomares) e em cinco locais (três no estado de Santa Catarina-SC e dois no estado do Paraná-PR). O experimento foi implantado seguindo o delineamento de blocos ao acaso, com 6 plantas por parcela, sendo que, em Santa Catarina (locais 1, 2 e 3), foram instalados 5 blocos por local, e, no Paraná (locais 4 e 5), o experimento foi implantado com 9 blocos por local, em espaçamento de $3 \mathrm{~m}$ x $3 \mathrm{~m}$, conforme tabela 1 .

Tabela 1. Localização e características dos locais nos municípios de Otacílio Costa (SC) e Telêmaco Borba (PR) onde foram instalados os experimentos com as progênies de Pinus taeda.

Table 1. Location and characteristics of locations in the Otacílio Costa (SC) and Telêmaco Borba (PR) municipalities where the progenies of Pinus taeda were planted.

\begin{tabular}{|c|c|c|c|c|}
\hline $\begin{array}{l}\text { Município - } \\
\text { estado }\end{array}$ & Fazenda & Local & Classificação do solo & $\begin{array}{c}\text { Sítio } \\
\text { classificação }\end{array}$ \\
\hline \multirow{3}{*}{$\begin{array}{l}\text { Otacílio Costa } \\
\text { - SC }\end{array}$} & Alexandrina & 1 & $\begin{array}{l}\text { Latossolo Bruno, alumínico, textura argilosa, } \\
\text { relevo suave ondulado e ondulado }\end{array}$ & Sítio 2 \\
\hline & Cerro Rico & 2 & $\begin{array}{l}\text { Cambissolo Húmico, alumínico, lépticos, textura } \\
\text { argilosa, relevo suave ondulado e ondulado }\end{array}$ & Sítio 1 \\
\hline & Bom Retiro & 3 & $\begin{array}{l}\text { Cambissolo Háplico, alumínico, lépticos, textura } \\
\text { muito argilosa, relevo suave ondulado e ondulado }\end{array}$ & Sítio 3 \\
\hline \multirow{2}{*}{$\begin{array}{l}\text { Telêmaco Borba } \\
\text { - PR }\end{array}$} & Imbauzinho & 4 & $\begin{array}{l}\text { Cambissolo Háplico, textura média, relevo } \\
\text { ondulado e forte ondulado }\end{array}$ & Sítio 1 \\
\hline & Invernadinha & 5 & $\begin{array}{l}\text { Neossolo Quartzarênico, textura arenosa e média } \\
\text { leve, relevo suave ondulado e forte ondulado }\end{array}$ & Sítio 2 \\
\hline
\end{tabular}


Segundo a classificação climática de Köppen, a região de Otacílio Costa (SC) caracteriza-se como Cfb (KLABIN, 2009) e a região de Telêmaco Borba (PR) encontra-se em uma região de transição climática entre Cfa e Cfb (KLABIN, 2011). Otacílio Costa apresenta temperaturas médias inferiores e um maior número de geadas do que Telêmaco Borba.

Aos 6 anos de idade, as progênies foram avaliadas quanto aos caracteres diâmetro $(\mathrm{em} \mathrm{cm}$, medido a $1,30 \mathrm{~m}$ de altura) e altura total (em m). Os solos de cada local foram analisados, identificados e classificados de acordo com a produtividade, para se avaliar a interação genótipos x ambientes. Ainda conforme a tabela 1, em Otacílio Costa (SC) os sítios foram classificados de 1 (melhor sítio) a 3 (pior sítio), e em Telêmaco Borba (PR) foram classificados em 1 (melhor sítio) e 2 (pior sítio).

As análises foram realizadas pelo procedimento de estimação de componentes de variância (Reml) e de predição de valores genéticos (Blup e Blup-Het), usando-se o software Selegen-Reml/Blup (RESENDE, 2002b). Para a avaliação dos componentes de variância, as variáveis foram avaliadas individualmente por local e em conjunto dos locais.

As variáveis foram analisadas usando-se a metodologia de modelo linear misto univariado aditivo do software Selegen-Reml/Blup, apresentado por Resende (2002b), consistindo da seguinte equação:

$$
y=X b+Z a+W c+e
$$

Em que: $y, b, a, c$ e $e=$ vetores de dados, dos efeitos de blocos (fixos), dos efeitos genéticos aditivos (aleatórios), de efeitos de parcela (aleatórios) e dos erros aleatórios, respectivamente;

$\mathrm{X}, \mathrm{Z}$ e W = matrizes de incidência para $\mathrm{b}$, a e c, respectivamente.

Foram determinadas as correlações entre os valores genéticos preditos entre os procedimentos Blup e Blup-Het para cada local e as correlações entre as variáveis diâmetro e altura para cada procedimento.

A estimativa das variáveis, considerando todos os locais, foi realizada no modelo linear misto, que inclui o efeito de interação genótipos x ambientes, o que resultou nos componentes de variância e predição dos valores genéticos, incluindo todos os locais, além de ser possível avaliar a interação através da adaptabilidade e estabilidade das progênies em relação aos diferentes locais. Utilizou-se o seguinte modelo genético-estatístico (RESENDE, 2007):

$$
y=X r+Z g+W p+\mathrm{Ti}+e
$$

Em que: $y, r, g, p, i$ e $e=$ vetores de dados, dos efeitos de blocos (fixos), dos efeitos genéticos aditivos (aleatórios), de efeitos de parcela (aleatórios), dos efeitos da interação genótipos x ambientes (aleatórios) e dos erros aleatórios, respectivamente;

$\mathrm{X}, \mathrm{Z}, \mathrm{W}$ e $\mathrm{T}=$ matrizes de incidência para $\mathrm{r}, \mathrm{g}, \mathrm{p}$ e i, respectivamente.

Nesses modelos, os componentes de média (Blup individual) resultam na classificação individual e por progênies, de acordo com os valores genéticos aditivos preditos.

Com os valores genéticos preditos, foram realizadas as correlações entre as duas variáveis estimadas por local e ainda a correlação entre locais (tomados dois a dois e em conjunto), para cada variável.

Foram avaliadas a produtividade, a estabilidade (através da média harmônica dos valores genéticos através dos locais - MHVG), a adaptabilidade (através da performance relativa dos valores genéticos em relação à média de cada local - PRVG) e a estabilidade e a adaptabilidade simultaneamente, através da média harmônica da performance relativa dos valores genéticos (MHPRVG), conforme Resende (2007).

Com os valores genéticos preditos, realizou-se uma simulação do ganho genético entre progênies, considerando uma intensidade de seleção de 20\% (30 progênies de um total de 150), por local e pelos critérios que levam em consideração todos os locais em conjunto, para comparação dos ganhos estimados e da nova média esperada da população. 


\section{RESULTADOS E DISCUSSÃO}

\section{Análise por local}

Os componentes de variância e parâmetros genéticos estimados por local para diâmetro e para altura são apresentados na tabela 2. Os maiores valores para variância genética aditiva, variância fenotípica, herdabilidades, acurácia, coeficientes de variação genética aditiva individual e genotípica entre progênies foram encontrados nos sítios mais produtivos (sítio 1) de cada região de plantio (locais 2 e 4).

Tabela 2. Componentes de variância estimados em cinco diferentes locais para a variável diâmetro (Dap) e altura $(\mathrm{H})$ em progênies de Pinus taeda nos municípios de Otacílio Costa (SC) e Telêmaco Borba (PR).

Table 2. Variance components estimated at five different locations for the variable diameter (Dap) and heigth (H) in progenies of Pinus taeda in the Otacílio Costa (SC) and Telêmaco Borba (PR) municipalities.

\begin{tabular}{|c|c|c|c|c|c|c|c|c|c|c|}
\hline \multirow{3}{*}{ Parâmetro } & \multicolumn{6}{|c|}{ Otacílio Costa - SC } & \multicolumn{4}{|c|}{ Telêmaco Borba - PR } \\
\hline & \multicolumn{2}{|c|}{ Local 1 / Sítio 2} & \multicolumn{2}{|c|}{ Local 2 / Sítio 1} & \multicolumn{2}{|c|}{ Local 3 / Sítio 3} & \multicolumn{2}{|c|}{ Local 4 / Sítio 1} & \multicolumn{2}{|c|}{ Local 5 / Sítio 2} \\
\hline & Dap & $\mathbf{H}$ & Dap & $\mathbf{H}$ & Dap & $\mathbf{H}$ & Dap & $\mathbf{H}$ & Dap & $\mathbf{H}$ \\
\hline$\sigma_{\mathrm{a}}^{2}$ & 1,416 & 0,110 & 3,954 & 0,438 & 2,303 & 0,237 & 5,901 & 1,434 & 2,696 & 0,472 \\
\hline$\sigma_{\text {parc }}^{2}$ & 0,149 & 0,106 & 0,038 & 0,103 & 0,321 & 0,099 & 0,157 & 0,208 & 0,195 & 0,195 \\
\hline$\sigma_{\mathrm{e}}^{2}$ & 6,234 & 0,978 & 6,000 & 0,770 & 4,978 & 0,718 & 7,554 & 1,026 & 6,433 & 1,155 \\
\hline$\sigma_{\mathrm{f}}^{2}$ & 7,800 & 1,195 & 9,993 & 1,312 & 7,603 & 1,055 & 13,613 & 2,670 & 9,326 & 1,822 \\
\hline $\mathrm{h}_{\mathrm{a}}^{2}$ & 0,181 & 0,092 & 0,395 & 0,333 & 0,303 & 0,225 & 0,433 & 0,537 & 0,289 & 0,259 \\
\hline & $\pm 0,036$ & $\pm 0,026$ & $\pm 0,053$ & $\pm 0,049$ & $\pm 0,047$ & $\pm 0,040$ & $\pm 0,042$ & $\pm 0,047$ & $\pm 0,034$ & $\pm 0,033$ \\
\hline $\mathrm{h}^{2}{ }_{\mathrm{aj}}$ & 0,185 & 0,101 & 0,397 & 0,362 & 0,316 & 0,248 & 0,438 & 0,582 & 0,295 & 0,290 \\
\hline$c^{2}$ parc & 0,019 & 0,088 & 0,003 & 0,078 & 0,042 & 0,094 & 0,011 & 0,078 & 0,020 & 0,107 \\
\hline $\mathrm{h}_{\mathrm{mp}}^{2}$ & 0,866 & 0,709 & 0,941 & 0,905 & 0,909 & 0,856 & 0,968 & 0,966 & 0,949 & 0,922 \\
\hline Acprog & 0,930 & 0,842 & 0,970 & 0,951 & 0,953 & 0,925 & 0,984 & 0,983 & 0,974 & 0,960 \\
\hline $\mathrm{h}^{2}{ }_{\mathrm{ad}}$ & 0,145 & 0,078 & 0,330 & 0,298 & 0,257 & 0,198 & 0,369 & 0,511 & 0,239 & 0,234 \\
\hline CVgi\% & 6,534 & 3,020 & 10,125 & 5,836 & 9,146 & 5,395 & 13,268 & 10,503 & 9,927 & 7,103 \\
\hline CVgp $\%$ & 3,267 & 1,510 & 5,062 & 2,918 & 4,573 & 2,697 & 6,634 & 5,251 & 4,963 & 3,551 \\
\hline $\mathrm{CVe} \%$ & 6,417 & 4,836 & 6,304 & 4,719 & 7,227 & 5,520 & 8,016 & 6,556 & 7,658 & 6,907 \\
\hline $\mathrm{CVr}$ & 0,509 & 0,312 & 0,803 & 0,618 & 0,632 & 0,488 & 0,827 & 0,800 & 0,648 & 0,514 \\
\hline PEV & 0,047 & 0,008 & 0,057 & 0,010 & 0,052 & 0,008 & 0,046 & 0,012 & 0,033 & 0,009 \\
\hline SEP & 0,217 & 0,089 & 0,240 & 0,101 & 0,228 & 0,092 & 0,215 & 0,109 & 0,184 & 0,095 \\
\hline Média geral & 18,209 & 11,003 & 19,639 & 11,341 & 16,595 & 9,037 & 18,308 & 11,404 & 16,542 & 9,677 \\
\hline
\end{tabular}

$\sigma_{\mathrm{a}}^{2}$ : variância genética aditiva; $\sigma_{\mathrm{parc}}^{2}$ : variância ambiental entre parcelas; $\sigma_{\mathrm{e}}^{2}$ : variância residual dentro de parcelas; $\sigma_{\mathrm{f}}^{2}$ : variância fenotípica individual; $\mathrm{h}_{\mathrm{a}}^{2}$ : herdabilidade individual no sentido restrito no bloco; $\mathrm{h}_{\mathrm{aj}}{ }_{\mathrm{aj}}$ : herdabilidade individual no sentido restrito, ajustada para os efeitos de parcela; $\mathrm{c}^{2}{ }_{\text {parc }}$ : coeficiente de determinação dos efeitos de parcela; $\mathrm{h}^{2}{ }_{\mathrm{mp}}$ : herdabilidade da média de progênie; Acprog: acurácia da seleção de progênies e genitores; $\mathrm{h}^{2}{ }_{\mathrm{ad}}$ : herdabilidade aditiva dentro da parcela; CVgi\%: coeficiente de variação genética aditiva individual; $\mathrm{CVgp} \%$ : coeficiente de variação genotípica entre progênies; CVe\%: coeficiente de variação residual; CVr: coeficiente de variação relativa; PEV: variância do erro de predição dos valores genotípicos de progênie; SEP: desvio padrão do valor genotípico predito de progênie.

Em Otacílio Costa, o local 1 (sítio 2), apesar de ter maior produtividade que o local 3 (sítio 3), apresentou menores estimativas de herdabilidades. Se, por um lado, os melhores sítios apresentaram melhores condições de desenvolvimento e da expressão do potencial genético das progênies, por outro lado, o pior sítio apresentou variação genética maior que o sítio intermediário, para as duas características avaliadas. Nesse caso, a menor qualidade de sítio pode ter interferido e limitado o desenvolvimento de algumas progênies, ocasionando maior variação.

Apesar da variabilidade existente entre locais, Otacílio Costa apresentou uma média geral para diâmetro pouco superior a Telêmaco Borba; quanto à altura, os dois locais foram praticamente iguais. Em termos médios e avaliando a variabilidade existente entre os locais, a diferença geográfica entre as regiões não ocasionou grandes interferências no desenvolvimento e na expressão potencial das progênies. A diferença existente entre locais se deve à interação genótipo x ambiente, refletida pelas necessidades ecológicas da espécie, características climáticas e dos solos. 
Comparando os resultados preditos em Otacílio Costa, a herdabilidade aditiva dentro de parcela e as herdabilidades individuais no sentido restrito do bloco e ajustada para os efeitos de parcela, o sítio 1 apresentou resultados aproximadamente duas vezes superior ao sítio 2 para diâmetro e cerca de 3,5 vezes superior para altura. Nesse caso, o sítio 2 demonstrou uma menor variação entre os genótipos, ocasionando uma redução nessas estimativas. Por outro lado, o sítio 3, apesar de apresentar os menores diâmetros e alturas, teve maiores variações e herdabilidades que o sítio 2, proporcionando, nesse caso, melhores condições para detectar a variação existente e, consequentemente, maiores possibilidades de ganhos genéticos por meio de seleção.

Em Telêmaco Borba, o sítio 1 apresentou herdabilidade aditiva dentro de parcela, individual no sentido restrito do bloco e ajustada para os efeitos de parcela cerca de 1,5 vezes superior para diâmetro e cerca de 2 vezes superior para altura, quando comparado ao sítio 2.

As herdabilidades aditivas médias de progênies, em cada região analisada, foram superiores nos melhores sítios. Considerando todos os locais, essas herdabilidades foram de elevada magnitude $(0,709$ a 0,968), tanto para diâmetro quanto para altura, conduzindo a altas acurácias $(0,842$ a 0,984$)$ na seleção de progênies, o que indica um expressivo controle genético. Nesse sentido, vale ressaltar que a mais importante função da herdabilidade no estudo genético do caráter métrico é o seu papel preditivo, expressando a confiança do valor fenotípico como um guia para o valor genético, ou o grau de correspondência entre o valor fenotípico e o valor genético (VENCOVSKY; BARRIGA, 1992; CORNELLIUS, 1994).

A baixa variância ambiental entre parcelas resultou em baixos coeficientes de determinação dos efeitos de parcela, explicando também a proximidade entre as herdabilidades individuais no sentido restrito, ajustadas e não ajustadas para os efeitos de parcela.

Os coeficientes de variação experimental foram de baixa magnitude (inferiores a 8,1\%), revelando uma boa precisão experimental, tanto para o diâmetro quanto para a altura. Os coeficientes de variação genética aditiva individual apresentaram valores superiores aos coeficientes de variação experimental (exceto para altura nos locais 1 e 3), demonstrando a variabilidade existente no experimento.

De maneira geral, apesar das diferenças observadas, os valores apresentaram-se parecidos nos cinco locais e para as duas variáveis avaliadas. Os resultados obtidos dos locais e das variáveis podem indicar uma semelhança na produtividade entre ambientes e uma boa correlação entre variáveis, tendo em vista que são os mesmos tratamentos. Devido à magnitude dos componentes de variância estimados por local, ganhos genéticos expressivos poderão ser obtidos em todos os sítios.

A heterogeneidade de variâncias residuais e herdabilidades individuais foram estimadas através do procedimento Blup-Het. A heterogeneidade de variâncias residuais para diâmetro e altura (Tabela 3) foi maior nos locais 2 e 4, ou seja, as variâncias residuais sofreram maior influência do sítio mais produtivo. Nesse caso, a heterogeneidade depende da expressão genética no ambiente, ocasionando desenvolvimento diferente das progênies em função das características do sítio.

Em Otacílio Costa (local 3 - sítio 3) e em Telêmaco Borba (local 5 - sítio 2), a produtividade inferior nos sítios, em termos de incremento diamétrico e em altura, expressou menor variação residual existente, com média das variâncias residuais por progênies inferior. Em relação à herdabilidade média de progênie (Tabela 3), observam-se as maiores herdabilidades nos melhores sítios (locais 2 e 4), por apresentarem melhores condições de expressão genética.

Com base nas variâncias residuais e nas herdabilidades, ocorreu uma grande variação entre progênies. Constata-se esse fato na tabela 3, com amplitudes elevadas (máximos e mínimos), bem como sua variância.

A tabela 4 demonstra, resumidamente, os valores para as 3 progênies com menores médias e maiores variâncias residuais obtidas e, para estas variâncias, as respectivas herdabilidades, considerando apenas o diâmetro. As progênies 24, 24019 e 470 tiveram variâncias residuais médias menores, ou seja, apresentaram valores mais próximos. As progênies 308, 41 e 10817 foram intermediárias, e as progênies 395, 108 e 10905 foram as que apresentaram maiores variâncias residuais médias. Quanto menor a variância existente entre locais, menor a variação observada para as progênies, ou seja, os resultados são mais próximos entre si do que quando comparadas às progênies com maiores variâncias. A elevada diferença existente entre as variâncias residuais mínimas e máximas demonstra a heterogeneidade de variâncias neste experimento. 
Tabela 3. Resultados médios, variância, desvio padrão, valores máximos e mínimos obtidos para a variância residual e herdabilidade das progênies de Pinus taeda por local, para o diâmetro (Dap) e altura (H), nos municípios de Otacílio Costa (SC) e Telêmaco Borba (PR).

Table 3. Results mean, variance, standard deviation, maximum and minimum values obtained for the residual variance and heritability of progenies of Pinus taeda per site, for the diameter (Dap) and height $(\mathrm{H})$, in the Otacílio Costa (SC) and Telêmaco Borba (PR) municipalities.

\begin{tabular}{|c|c|c|c|c|c|c|c|c|c|c|}
\hline \multirow{3}{*}{ Patrâmetro } & \multicolumn{6}{|c|}{ Otacílio Costa - SC } & \multicolumn{4}{|c|}{ Telêmaco Borba - PR } \\
\hline & \multicolumn{2}{|c|}{ Local 1 / Sítio 2} & \multicolumn{2}{|c|}{ Local 2 / Sítio 1} & \multicolumn{2}{|c|}{ Local 3 / Sítio 3} & \multicolumn{2}{|c|}{ Local 4 / Sítio 1} & \multicolumn{2}{|c|}{ Local 5 / Sítio 2} \\
\hline & Dap & $\mathbf{H}$ & Dap & $\mathbf{H}$ & Dap & $\mathbf{H}$ & Dap & $\mathbf{H}$ & Dap & $\mathbf{H}$ \\
\hline \multicolumn{11}{|c|}{ Variância residual das progênies } \\
\hline Média & 7,221 & 1,027 & 8,928 & 1,064 & 6,577 & 0,867 & 11,856 & 2,022 & 8,330 & 1,446 \\
\hline Variância & 9,561 & 0,289 & 14,157 & 0,351 & 12,274 & 0,284 & 14,548 & 0,634 & 8,851 & 0,367 \\
\hline Desvio padrão & 3,092 & 0,537 & 3,762 & 0,593 & 3,503 & 0,533 & 3,814 & 0,796 & 2,975 & 0,606 \\
\hline Máximo & 16,648 & 3,272 & 26,413 & 3,844 & 22,577 & 2,993 & 25,062 & 5,304 & 22,961 & 4,005 \\
\hline Mínimo & 2,172 & 0,279 & 1,876 & 0,281 & 2,470 & 0,176 & 5,213 & 0,702 & 2,802 & 0,441 \\
\hline \multicolumn{11}{|c|}{ Herdabilidade das progênies } \\
\hline Média & 0,215 & 0,113 & 0,449 & 0,399 & 0,363 & 0,282 & 0,471 & 0,604 & 0,320 & 0,299 \\
\hline Variância & 0,008 & 0,002 & 0,026 & 0,020 & 0,018 & 0,013 & 0,016 & 0,032 & 0,008 & 0,010 \\
\hline Desvio padrão & 0,091 & 0,047 & 0,161 & 0,143 & 0,134 & 0,116 & 0,128 & 0,179 & 0,094 & 0,100 \\
\hline Máximo & 0,529 & 0,267 & 1,000 & 0,886 & 0,684 & 0,709 & 0,862 & 1,000 & 0,734 & 0,625 \\
\hline Mínimo & 0,082 & 0,032 & 0,144 & 0,108 & 0,098 & 0,075 & 0,221 & 0,244 & 0,113 & 0,109 \\
\hline
\end{tabular}

Tabela 4. Variâncias residuais e herdabilidades individuais das progênies de Pinus taeda com menores, intermediárias e maiores variâncias na análise conjunta e por local para diâmetro, avaliados nos municípios de Otacílio Costa (SC) e Telêmaco Borba (PR).

Table 4. Residual variances and heritability individuals of progenies of Pinus taeda with minor, intermediate and higher variances in the combined analysis and location for diameter, evaluated in the Otacílio Costa (SC) and Telêmaco Borba (PR) municipalities.

\begin{tabular}{|c|c|c|c|c|c|c|c|c|}
\hline \multirow[b]{2}{*}{ Classificação } & \multirow[b]{2}{*}{ Progênie } & \multicolumn{3}{|c|}{ Otacílio Costa - SC } & \multicolumn{2}{|c|}{ Telêmaco Borba - PR } & \multirow[b]{2}{*}{ Média } & \multirow[b]{2}{*}{ Variância } \\
\hline & & $\begin{array}{c}\text { Local } 1 / \\
\text { Sítio } 2 \\
\end{array}$ & $\begin{array}{c}\text { Local 2 / } \\
\text { Sítio } 1 \\
\end{array}$ & $\begin{array}{c}\text { Local } 3 \text { / } \\
\text { Sítio } 3 \\
\end{array}$ & $\begin{array}{c}\text { Local } 4 \text { / } \\
\text { Sítio } 1 \\
\end{array}$ & $\begin{array}{c}\text { Local } 5 \text { / } \\
\text { Sítio } 2 \\
\end{array}$ & & \\
\hline \multicolumn{9}{|c|}{ Variâncias residuais } \\
\hline \multirow{3}{*}{$\begin{array}{l}\text { Menores } \\
\text { variâncias }\end{array}$} & 24 & 6,489 & 5,524 & 5,287 & 6,071 & 6,223 & 5,919 & 0,248 \\
\hline & 24019 & 8,735 & 10,714 & 8,484 & 9,368 & 9,283 & 9,317 & 0,747 \\
\hline & 470 & 7,205 & 4,564 & 6,727 & 5,917 & 5,520 & 5,987 & 1,069 \\
\hline \multirow{3}{*}{$\begin{array}{l}\text { Variâncias } \\
\text { intermediárias }\end{array}$} & 308 & 3,002 & 10,596 & 3,426 & 7,577 & 3,780 & 5,676 & 10,907 \\
\hline & 41 & 8,904 & 13,075 & 3,805 & 8,147 & 9,355 & 8,657 & 10,966 \\
\hline & 10817 & 5,358 & 11,994 & 5,754 & 11,852 & 6,626 & 8,317 & 11,050 \\
\hline \multirow{3}{*}{$\begin{array}{l}\text { Maiores } \\
\text { variâncias }\end{array}$} & 395 & 7,277 & 25,596 & 7,396 & 17,685 & 13,432 & 14,277 & 59,201 \\
\hline & 108 & 3,130 & 6,696 & 9,064 & 23,424 & 8,786 & 10,220 & 60,105 \\
\hline & 10905 & 4,016 & 26,413 & 13,094 & 17,281 & 22,961 & 16,753 & 76,936 \\
\hline \multicolumn{9}{|c|}{ Herdabilidades individuais } \\
\hline \multirow{3}{*}{$\begin{array}{l}\text { Menores } \\
\text { variâncias }\end{array}$} & 24 & 0,202 & 0,603 & 0,372 & 0,766 & 0,380 & 0,464 & 0,048 \\
\hline & 24019 & 0,153 & 0,336 & 0,245 & 0,536 & 0,265 & 0,307 & 0,020 \\
\hline & 470 & 0,183 & 0,707 & 0,302 & 0,781 & 0,422 & 0,479 & 0,066 \\
\hline \multirow{3}{*}{$\begin{array}{l}\text { Variâncias } \\
\text { intermediárias }\end{array}$} & 308 & 0,403 & 0,340 & 0,532 & 0,640 & 0,579 & 0,499 & 0,015 \\
\hline & 41 & 0,150 & 0,280 & 0,489 & 0,603 & 0,263 & 0,357 & 0,033 \\
\hline & 10817 & 0,241 & 0,303 & 0,346 & 0,437 & 0,359 & 0,337 & 0,005 \\
\hline \multirow{3}{*}{$\begin{array}{l}\text { Maiores } \\
\text { variâncias }\end{array}$} & 395 & 0,182 & 0,148 & 0,277 & 0,305 & 0,188 & 0,220 & 0,004 \\
\hline & 108 & 0,389 & 0,512 & 0,231 & 0,235 & 0,279 & 0,329 & 0,014 \\
\hline & 10905 & 0,313 & 0,144 & 0,164 & 0,312 & 0,113 & 0,209 & 0,009 \\
\hline
\end{tabular}


Quanto às herdabilidades individuais por progênie apresentadas na tabela 4, ocorreu uma grande variação entre progênies e até mesmo dentro de progênies entre locais. Nesse caso, o uso de uma única herdabilidade média para estimar o valor genético, como feito pelo procedimento Blup, pode ocasionar erros, subestimando ou superestimando os valores genéticos para determinadas progênies. A utilização do procedimento Blup-Het, com herdabilidades individuais por progênie, resulta em uma melhor estimativa dos valores genéticos e, consequentemente, uma redução do erro quadrático médio e aumento da acurácia.

As correlações entre os valores genéticos preditos dos procedimentos Blup e Blup-Het por local e caráter (Tabela 5) foram elevadas, tanto para diâmetro quanto para altura, o que conduz praticamente à seleção dos mesmos genótipos (nesse caso, os dois procedimentos podem ser utilizados como critério de seleção).

As correlações genéticas entre diâmetro e altura pelos procedimentos Blup e Blup-Het, de forma similar foram altas, sendo positivas e superiores a $72 \%$ em todos os locais (Tabela 5). Não ocorreu uma tendência de aumento ou diminuição das correlações em função da qualidade do sítio. Correlações altas entre esses caracteres também foram encontradas por Paludzyszyn Filho et al. (2002), sendo de 0,94 aos 84 meses de idade, e por Duda (2003), variando de 0,80 a 0,94, de acordo com o local. Correlações elevadas e positivas sugerem que a seleção para um caráter deve levar a consistentes respostas indiretas nos outros caracteres (BLADA, 2003). A avaliação aos seis anos, apesar de precoce, não se torna problemática, segundo alguns pesquisadores que também avaliaram Pinus taeda em idades semelhantes, pois constataram alta correlação com plantas de idades avançadas. Paludzyszyn Filho et al. (2003) avaliaram progênies de Pinus taeda aos 16 e 84 meses de idade e encontraram correlações genéticas entre idades de alta magnitude para diâmetro, não havendo necessidade de se esperar todo o ciclo de produção para a avaliação genética. Com essas correlações elevadas, o uso do diâmetro (por ser uma característica de maior facilidade de obtenção e com menor erro de medição) deve ser preferido na seleção, propiciando ganho indireto para altura.

Tabela 5. Correlações dos procedimentos Blup x Blup-Het para diâmetro e altura e correlações diâmetro $\mathrm{x}$ altura para os referidos procedimentos, por local, entre progênies de Pinus taeda, aos 6 anos de idade, avaliados nos municípios de Otacílio Costa (SC) e Telêmaco Borba (PR).

Table 5. Correlations Blup x Blup-Het for diameter and height and correlations diameter $\mathrm{x}$ height for the procedures Blup and Blup-Het by place, between progenies of Pinus taeda, at six years old, evaluated in the Otacílio Costa (SC) and Telêmaco Borba (PR) municipalities.

\begin{tabular}{lccccc}
\hline \multirow{2}{*}{ Município } & \multirow{2}{*}{ Local } & \multicolumn{2}{c}{ Correlação Blup x Blup-Het } & \multicolumn{2}{c}{ Correlação Diâmetro x Altura } \\
\cline { 3 - 6 } & Local 1 / Sítio 2 & 0,989 & 0,979 & 0,728 & 0,733 \\
\multirow{2}{*}{ Otacílio Costa - SC } & Local 2 / Sítio 1 & 0,989 & 0,992 & 0,778 & 0,772 \\
& Local 3 / Sítio 3 & 0,989 & 0,986 & 0,842 & 0,834 \\
\hline \multirow{2}{*}{ Telêmaco Borba - PR } & Local 4 / Sítio 1 & 0,999 & 0,999 & 0,888 & 0,887 \\
& Local 5 / Sítio 2 & 0,997 & 0,998 & 0,856 & 0,851 \\
\hline
\end{tabular}

Nessas condições, apesar de os dois procedimentos conduzirem aos mesmos resultados práticos, o procedimento Blup-Het deve ser preferido para estimação dos parâmetros genéticos e seleção, pois, com a heterogeneidade de variâncias residuais, a estimativa de herdabilidade individual por progênie se torna mais precisa do que utilizando a herdabilidade média do local.

\section{Análise conjunta}

Os componentes de variância através da análise conjunta para diâmetro e para altura (Tabela 6) apresentaram baixas variâncias ambientais entre parcelas e da interação genótipos x ambientes, resultando em baixos coeficientes de determinação dos efeitos de parcela e dos efeitos da interação. As herdabilidades das médias das progênies apresentaram altos valores, conduzindo a expressivas acurácias seletivas, para ambos os caracteres. Os coeficientes de variação residual e genética livre da interação genótipos x ambientes, de forma similar, também foram de baixa magnitude.

As diferenças obtidas entre os componentes de variância através das análises conjuntas e as análises individuais se devem ao fato de se analisar todo o experimento em conjunto, obtendo-se componentes de variância médios entre os locais. 
As correlações genéticas entre o comportamento das progênies através dos locais (Tabela 7), tomadas duas a duas e em grupo, permitem inferir que as correlações foram de menor magnitude para o caráter altura (de 0,179 a 0,803). Duda (2003) encontrou correlações de alta magnitude avaliando três locais, analisados dois a dois, sendo que para o diâmetro as correlações variaram entre 0,85 e 0,94 , e para a altura variaram entre 0,81 e 0,92 . Hodge et al. (2003) encontraram uma interação praticamente nula entre genótipo e ambiente, sugerindo uma correlação próxima a 1. Os dados obtidos para altura apresentaram menor precisão de mensuração em comparação ao diâmetro, sendo, portanto, mais sujeitos aos erros de medição. Acrescenta-se o fato de que o diâmetro é mais correlacionado com o volume do que a altura, pois o volume é uma função do quadrado do diâmetro. Devido a esses motivos, os resultados referentes ao diâmetro, neste caso, são mais relevantes.

Tabela 6. Componentes de variância estimados através da análise conjunta para diâmetro e altura em progênies de Pinus taeda, nos municípios de Otacílio Costa (SC) e Telêmaco Borba (PR).

Table 6. Variance components estimated in the combined analysis for diameter and height in progenies of Pinus taeda, in the Otacilio Costa (SC) and Telemaco Borba (PR) municipalities.

\begin{tabular}{lcc}
\hline Parâmetro & Diâmetro & Altura \\
\hline$\sigma_{\mathrm{g}}^{2}$ & 0,569 & 0,091 \\
$\sigma_{\text {parc }}^{2}$ & 0,192 & 0,151 \\
$\sigma^{2}$ int & 0,242 & 0,068 \\
$\sigma^{2}$ & 9,040 & 1,447 \\
$\sigma_{\mathrm{e}}^{2}$ & 10,046 & 1,758 \\
$\mathrm{~h}_{\mathrm{f}}^{2}$ & $0,056 \pm 0,004$ & $0,052 \pm 0,004$ \\
$\mathrm{c}^{2}{ }_{\text {parc }}$ & 0,019 & 0,086 \\
$\mathrm{c}^{2}{ }_{\text {int }}$ & 0,024 & 0,038 \\
$\mathrm{~h}^{2}{ }_{\text {mg }}$ & 0,905 & 0,849 \\
Acgen & 0,951 & 0,921 \\
$\mathrm{CVgi \%}$ & 4,252 & 2,875 \\
$\mathrm{CVe} \%$ & 7,344 & 5,970 \\
Média geral & 17,750 & 10,503 \\
\hline
\end{tabular}

$\sigma_{\mathrm{g}}^{2}$ : variância genética aditiva; $\sigma_{\text {parc }}^{2}$ variância ambiental entre parcelas; $\sigma_{\text {int }}^{2}$ variância da interação genótipos $\mathrm{x}$ ambientes; $\sigma_{\mathrm{e}}^{2}$ : variância residual; $\sigma_{\mathrm{f}}^{2}$ : variância fenotípica individual; $\mathrm{h}_{\mathrm{g}}{ }_{\mathrm{g}}$ : herdabilidade individual no sentido restrito, ou seja, dos efeitos aditivos; c $^{2}$ parc: coeficiente de determinação dos efeitos de parcela; $\mathrm{c}^{2}$ int: coeficiente de determinação dos efeitos da interação genótipos $\mathrm{x}$

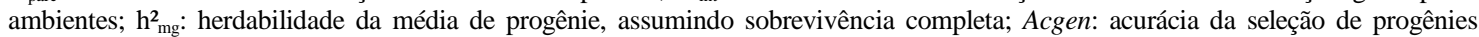
assumindo sobrevivência completa; CVgi\%: coeficiente de variação genética aditiva individual; CVe\%: coeficiente de variação residual.

Tabela 7. Correlações genéticas entre o comportamento das progênies de Pinus taeda através dos locais tomados dois a dois por local, entre locais e em grupo, avaliados nos municípios de Otacílio Costa (SC) e Telêmaco Borba (PR).

Table 7. Genetic correlations between progenies of Pinus taeda through sites through sites taken two by two per site, between sites and group, evaluated in the Otacílio Costa (SC) and Telêmaco Borba (PR) municipalities.

\begin{tabular}{lccc}
\hline Correlação por município & Combinação entre locais & Diâmetro & Altura \\
\hline \multirow{2}{*}{ Otacílio Costa - SC } & Locais 1 e 2 & 0,376 & 0,179 \\
& Locais 1 e 3 & 0,547 & 0,384 \\
Telêmaco Borba - PR & Locais 2 e 3 & 0,941 & 0,742 \\
& Locais 4 e 5 & 0,791 & 0,691 \\
& Locais 1 e 4 & 0,356 & 0,305 \\
& Locais 1 e 5 & 0,432 & 0,389 \\
Otacílio Costa - SC x Telêmaco Borba - PR & Locais 2 e 4 & 0,829 & 0,740 \\
& Locais 2 e 5 & 0,808 & 0,803 \\
& Locais 3 e 4 & 0,747 & 0,514 \\
& Locais 3 e 5 & 0,779 & 0,683 \\
& Locais 2, 3, 4 e 5 & 0,799 & 0,666 \\
\hline
\end{tabular}


Para o caráter diâmetro, constata-se que quatro $(2,3,4$ e 5$)$ dos cinco ambientes foram altamente correlacionados, com uma correlação de 0,799 na análise conjunta dos quatro ambientes, simultaneamente. Isso significa uma coincidência de 8 progênies dentre as 10 melhores selecionadas em cada local. Nesses ambientes, as correlações entre locais tomados dois a dois variaram de 0,747 a 0,941.

A avaliação de progênies em diferentes locais é fundamental em testes genéticos, pois diferentes resultados podem ser obtidos de acordo com cada local. Falkenhagen (1989) encontrou forte interferência do sítio na correlação entre altura e diâmetro, avaliando experimentos com Pinus elliottii na África do Sul, em diferentes regiões climáticas. Paludzyszyn Filho et al. (2001) avaliaram progênies de Pinus taeda em quatro diferentes locais e sugerem que o programa de melhoramento deve ser específico por local. Por outro lado, Duda (2003) sugere um único programa de melhoramento de Pinus taeda para três diferentes locais estudados, devido à alta correlação genética. A opção pela seleção por local ou conjunta depende diretamente dos genótipos selecionados em cada local, suas herdabilidades e correlações genéticas. Correlações acima de $2 / 3$ ou 0,67 são consideradas altas e indicam que um só programa de melhoramento atende satisfatoriamente a todos os locais, simultaneamente (RESENDE, 2002a). Neste estudo, a análise considerando todos os locais também foi alta, podendo ser utilizada a mesma seleção para o programa de melhoramento. Considerando todos os locais, a correlação foi de 0,701 , e apesar de ser inferior ao valor obtido nos quatro ambientes, também foi alta.

Verifica-se ainda, na tabela 7 , que o local 1 apresentou correlações genéticas de menores magnitudes com os demais locais, em média em torno de 0,43 para diâmetro. Entretanto, tal local é representativo de uma área de plantio muito pequena. Mesmo assim, há uma coincidência de quatro progênies dentre as 10 melhores selecionadas em cada local.

Na tabela 8 estão descritos os valores médios, amplitudes e variâncias para diâmetro, considerando todos os locais em conjunto e todas as progênies.

Tabela 8. Variância residual e herdabilidade para diâmetro considerando todos os locais em conjunto e todas as progênies de Pinus taeda, avaliados nos municípios de Otacílio Costa (SC) e Telêmaco Borba (PR).

Table 8. Residual variance and heritability for diameter considering all sites together and all the progenies of Pinus taeda, evaluated in the Otacílio Costa (SC) and Telêmaco Borba (PR) municipalities.

\begin{tabular}{lcc}
\hline \multirow{2}{*}{ Parâmetro } & \multicolumn{2}{c}{ Diâmetro } \\
\cline { 2 - 3 } & Variância residual & $\mathbf{h}^{2}$ individual \\
\hline Média & 8,821 & 0,240 \\
Máximo & 17,486 & 0,371 \\
Mínimo & 5,129 & 0,123 \\
Variância & 4,165 & 0,002 \\
\hline
\end{tabular}

Avaliando a heterogeneidade das variâncias residuais e as herdabilidades médias, observa-se uma elevada diferença nas amplitudes entre progênies. Apesar de a seleção através do valor médio conduzir a resultados semelhantes aos obtidos pelas herdabilidades individuais, a estimação dos parâmetros considerando cada progênie individualmente aumenta a eficiência na predição dos valores genéticos. Tais valores também podem ser observados na tabela 9, onde são apresentadas as 3 progênies com menores variâncias residuais (10278, 308 e 10933), 3 progênies intermediárias (progênies 41, 24007 e 10817) e 3 progênies com maiores variâncias residuais (21018, 101 e 10905). Assim, como observado nas análises por local, a análise conjunta também demonstra a heterogeneidade das variâncias residuais e a variação existente entre as herdabilidades médias por progênie, porém, por considerar todos os locais em conjunto, essa variância é menor. Devido a essa heterogeneidade, o procedimento Blup-Het poderá conduzir a estimativas mais precisas dos valores genéticos.

As herdabilidades de maiores magnitudes proporcionam a expressão genética de indivíduos superiores, em função do maior controle genético. Nesse sentido, considerando a seleção das 30 melhores progênies, estas apresentaram resultados comuns para diâmetro e altura em 23 progênies pelo critério de produtividade e estabilidade (MHVG), 21 progênies pelos valores genotípicos adicionados da interação média nos vários locais $(\mathrm{u}+\mathrm{g}+\mathrm{gem})$ e 22 progênies para os critérios de produtividade e adaptabilidade 
(PRVG) e produtividade, estabilidade e adaptabilidade (MHPRVGxMG). Essa elevada coincidência (superior a 2/3) era esperada, conforme discutido nas análises de correlações e considerando todos os ambientes em conjunto.

A seleção das 30 melhores progênies para cada característica entre os critérios utilizados apresentou pelo menos 27 progênies em comum, neste caso, entre o critério de produtividade e estabilidade (MHVG) com os demais. Os outros critérios apresentaram entre 28 a 30 progênies em comum. Assim, a seleção pelo valor genético resulta, neste caso, também na seleção para adaptabilidade e estabilidade, apresentando, assim, as melhores produtividades e a menor interação entre genótipos $\mathrm{x}$ ambientes.

A avaliação da produtividade, adaptabilidade e estabilidade deve ser realizada tendo como foco melhorar e aumentar a produtividade, reduzindo custos na implantação futura. Isso irá depender do comportamento das progênies em cada sítio para, somente após esta avaliação, definir quais serão os melhores critérios de seleção. Sun (2004) avaliou a adaptabilidade e estabilidade de famílias introduzidas de Pinus taeda na província de Fujian, na China, encontrando diferença entre famílias e entre locais, e observou que um dos locais era mais propício ao crescimento em diâmetro e volume, outro local apresentou maior crescimento em altura e um terceiro local apresentou redução em todas as características avaliadas. O autor pôde identificar famílias específicas por local para produtividade e, no conjunto de locais, algumas selecionadas com foco em adaptabilidade e estabilidade. Porém, avaliando os experimentos instalados no Paraná e em Santa Catarina, as diferenças entre locais foram baixas, conduzindo praticamente à seleção dos melhores genótipos para ambos os locais.

Tabela 9. Variância residual e herdabilidade para diâmetro, considerando as 3 progênies de Pinus taeda com menores, médias e maiores variâncias residuais, em 5 locais avaliados, nos municípios de Otacílio Costa (SC) e Telêmaco Borba (PR).

Table 9. Residual variance and heritability for diameter, considering the three progenies of Pinus taeda with lower, middle and higher residual variances, measured in 5 sites, in the Otacílio Costa (SC) and Telêmaco Borba (PR) municipalities.

\begin{tabular}{lccc}
\hline Ranking em relação às variâncias & Progênie & Variância residual & $\mathbf{h}^{\mathbf{2}}$ individual \\
\hline \multirow{3}{*}{ Menores variâncias } & 10278 & 5,129 & 0,371 \\
& 308 & 5,558 & 0,347 \\
& 10933 & 5,569 & 0,346 \\
\hline \multirow{3}{*}{ Variâncias médias } & 41 & 8,462 & 0,240 \\
& 24007 & 8,507 & 0,239 \\
& 10817 & 8,517 & 0,239 \\
\hline \multirow{2}{*}{ Maiores variâncias } & 21018 & 15,124 & 0,141 \\
& 101 & 16,201 & 0,132 \\
& 10905 & 17,486 & 0,123 \\
\hline
\end{tabular}

Para Tambarussi et al. (2010), na seleção de plantas superiores em programas de melhoramento, é importante compreender as relações entre herdabilidades e suas correlações, além da origem da semente e do sistema reprodutivo da espécie. Levando em consideração os resultados obtidos para os componentes de variância, as altas correlações genéticas entre os locais e entre as variáveis, bem como a semelhança das progênies na avaliação da estabilidade, adaptabilidade e produtividade, constatou-se que houve baixa interação genótipos x ambientes entre os locais avaliados. Para Cruz et al. (2004), a interação genótipos x ambientes ocorre quando há respostas diferentes dos genótipos avaliados em relação à variação existente no ambiente. Nunes et al. (2002) relataram que a resposta correlacionada pela seleção em um ambiente e ganho em outro sempre foi inferior ao ganho da seleção direta no local, quando há interação significativa.

Nessa linha, o programa de melhoramento genético de Pinus taeda pode ser conduzido em apenas um dos locais, visando atender as regiões de plantio da empresa, visto que a interação genótipos $\mathrm{x}$ ambientes é de baixa magnitude, corroborada pelos baixos coeficientes de determinação dos efeitos da interação genótipos x ambientes e pelas semelhanças obtidas por critérios de adaptabilidade, estabilidade e produtividade, reduzindo os custos dos programas de melhoramento e selecionando os melhores genótipos considerando todos os ambientes em conjunto. 


\section{Estimativa do ganho de seleção}

As estimativas dos ganhos de seleção por local entre progênies são apresentadas na tabela 10, e os ganhos com seleção das progênies considerando todo o experimento estão contidos na tabela 11.

As estimativas do ganho de seleção entre progênies e da média esperada por local revelaram ganhos que variaram ao longo dos locais, sendo de 6,6\% a 16,8\% para o diâmetro e de $2 \%$ a até $12,4 \%$ para altura. Em todos os locais e variáveis, o procedimento Blup-Het apresentou estimativas pouco maiores do ganho genético do que o procedimento Blup, que se mostrou mais conservador. A diferença existente está no fato de que o procedimento Blup utiliza uma única herdabilidade para todas as estimativas, enquanto que o Blup-Het utiliza uma herdabilidade individual para cada progênie. No local 1 , o Blup-Het apresentou estimativa maior que o Blup de 13,6\% para o diâmetro e de 16,5\% para a altura. Nos demais locais, a diferença dos procedimentos variou entre $1,9 \%$ e $8,4 \%$. A diferença no uso da herdabilidade por procedimento pode selecionar diferentes progênies, porém, tanto para o diâmetro quanto para a altura, o número de progênies selecionadas pelos procedimentos Blup e Blup-Het variou entre 28 e 30 coincidências, ou seja, nesse caso os dois procedimentos conduzem a resultados muito próximos na seleção.

Tabela 10. Valor médio predito, ganho de seleção (GS) e ganho de seleção em porcentagem (GS\%) para diâmetro e altura, por local e para os procedimentos Blup e Blup-Het, considerando uma intensidade de $20 \%$ de seleção entre progênies de Pinus taeda, avaliados nos municípios de Otacílio Costa (SC) e Telêmaco Borba (PR).

Table 10. Average predicted, selection gain (GS) and selection gain in percentage (GS\%) for diameter and height, for location and for procedures Blup and Blup-Het, considering an intensity of $20 \%$ of selection in progenies of Pinus taeda, evaluated in the Otacílio Costa (SC) and Telêmaco Borba (PR) municipalities.

\begin{tabular}{lccccc}
\hline \multirow{2}{*}{ Local } & \multirow{2}{*}{ Parâmetro } & \multicolumn{2}{c}{ Diâmetro } & \multicolumn{2}{c}{ Altura } \\
\cline { 2 - 5 } Local 1 / Sítio 2 & Média Esperada & 19,417 & 19,581 & 11,223 & 11,259 \\
& GS & 1,207 & 1,371 & 0,219 & 0,255 \\
& GS\% & 6,629 & 7,528 & 1,989 & 2,317 \\
\hline \multirow{2}{*}{ Local 2 / Sítio 1 } & Média Esperada & 21,973 & 22,084 & 12,017 & 12,050 \\
& GS & 2,334 & 2,445 & 0,675 & 0,708 \\
& GS\% & 11,883 & 12,448 & 5,956 & 6,239 \\
\hline \multirow{2}{*}{ Local 3 / Sítio 3 } & Média Esperada & 18,163 & 18,276 & 9,445 & 9,480 \\
& GS & 1,568 & 1,681 & 0,408 & 0,442 \\
& GS\% & 9,447 & 10,127 & 4,516 & 4,896 \\
\hline \multirow{2}{*}{ Local 4 / Sítio 1 } & Média Esperada & 21,322 & 21,393 & 12,794 & 12,820 \\
& GS & 3,014 & 3,085 & 1,390 & 1,416 \\
Local 5 / Sítio 2 & GS\% & 16,462 & 16,848 & 12,185 & 12,417 \\
& Média Esperada & 18,408 & 18,447 & 10,324 & 10,336 \\
\hline
\end{tabular}

As estimativas do ganho de seleção e da média esperada pelos critérios de produtividade e estabilidade (MHVG), produtividade e adaptabilidade (PRVG*MG), produtividade, estabilidade e adaptabilidade (MHPRVG*MG) e pelos valores genotípicos (u+g+gem) (Tabela 11) apresentaram ganhos esperados inferiores aos obtidos por local, exceto no caso da variável altura no local 1. Essa menor estimativa está associada à seleção das progênies que apresentam bons desempenhos em ambos os ambientes, mas não sendo, necessariamente, as melhores de cada ambiente. A interação reduz a correlação entre os valores genotípicos e fenotípicos, ocasionando também uma diminuição dos ganhos genéticos com a seleção (NUNES, 2000). Essa menor estimativa era esperada, tendo em vista que o maior ganho é obtido nos casos de seleção direta para a característica de interesse e para o local específico. Os ganhos considerando esses critérios variaram entre 5,4\% e 5,9\% para o diâmetro e entre 2,7\% e 3,5\% para 
a altura. A semelhança no ganho genético esperado, as correlações entre locais elevadas e os genótipos selecionados pelos critérios que envolvem a produtividade, estabilidade e a adaptabilidade reforçam a sugestão de que um único programa de seleção pode ser conduzido.

Tabela 11. Valor médio predito, ganho de seleção (GS) e ganho de seleção em porcentagem (GS\%) para as variáveis diâmetro e altura, considerando uma intensidade de $20 \%$ de seleção entre progênies de Pinus taeda, pelos critérios de produtividade e estabilidade (MHVG), produtividade e adaptabilidade ( $\mathrm{PRVG} * \mathrm{MG}$ ), produtividade, estabilidade e adaptabilidade (MHPRVG*MG) e pelos valores genotípicos $(\mathrm{u}+\mathrm{g}+\mathrm{gem})$, nos municípios de Otacílio Costa (SC) e Telêmaco Borba (PR).

Table 11. Average predicted, selection gain (GS) and selection gain in percentage (GS\%) for the diameter and height, assuming a 20\% intensity of selection among progenies of Pinus taeda, by the criteria of productivity and stability (MHVG), productivity and adaptability (PRVG*MG), productivity, stability and adaptability (MHPRVG*MG) and the genotypic values $(\mathrm{u}+\mathrm{g}+\mathrm{gem})$, in the Otacílio Costa (SC) and Telêmaco Borba (PR) municipalities.

\begin{tabular}{lccc}
\hline Critério & Parâmetro & Diâmetro & Altura \\
\hline \multirow{2}{*}{ Produtividade e estabilidade (MHVG) } & Média Esperada & 18,800 & 10,788 \\
& GS & 1,050 & 0,284 \\
& GS\% & 5,913 & 2,708 \\
\hline \multirow{2}{*}{ Produtividade e adaptabilidade (PRVG*MG) } & Média Esperada & 18,727 & 10,876 \\
& GS & 0,977 & 0,373 \\
& GS\% & 5,504 & 3,548 \\
\hline \multirow{2}{*}{ Produtividade, estabilidade e adaptabilidade } & Média Esperada & 18,722 & 10,873 \\
(MHPRVG*MG) & GS & 0,972 & 0,370 \\
& GS\% & 5,473 & 3,520 \\
\hline \multirow{2}{*}{ Valores genotípicos (u+g+gem) } & Média Esperada & 18,732 & 10,877 \\
& GS & 0,981 & 0,374 \\
& GS\% & 5,529 & 3,557 \\
\hline
\end{tabular}

\section{CONCLUSÕES}

- A similaridade dos ambientes e dos componentes de variância, as altas correlações genéticas e semelhanças das progênies selecionadas nos critérios de adaptabilidade, estabilidade e produtividade, que resultou em uma interação genótipos $\mathrm{x}$ ambientes de baixa magnitude, sugerem que o programa de melhoramento genético pode ser conduzido em apenas um dos locais.

- Apesar de apresentarem resultados muito próximos em função das altas correlações genéticas entre os caracteres altura e diâmetro, o procedimento Blup-Het deve ser utilizado na estimativa de parâmetros genéticos, pois, com a heterogeneidade de variâncias residuais, a estimativa baseada na herdabilidade individual por progênie é mais precisa do que a estimativa utilizando a herdabilidade média do local.

\section{REFERÊNCIAS}

ASSOCIAÇÃO BRASILEIRA DE PRODUTORES DE FLORESTAS PLANTADAS (ABRAF). Anuário estatístico da ABRAF 2011 ano base 2010. Brasília: ABRAF, 2011. 130 p.

BLADA, I. Genetic variation in stone pine half-sib progenies. In: McKINLEY, C.R. (Ed.), Proc. 27 ${ }^{\text {th }}$ Southern Forest Tree Improvement Conference, Stillwater, Oklahoma, USA, p. 85 - 95, 2003.

CORNELLIUS, J. Heretabilities and additive genetic coefficients of variation in forest trees. Canadian Journal of Forestry Research, v. 24, p. 371 - 379, 1994.

CRUZ, C. D.; REGAZZI, A. J.; CARNEIRO, P. C. S. Modelos biométricos aplicados ao melhoramento genético. v. 1, 3. ed., Viçosa: UFV, 2004. 480 p. 
DEMERITT, M. E. JR.; GARRET, P. W. Adaptation of eastern white pine provenances to planting sites. Research Paper NE-703, USDA Forest Service, Northeastern Forest Experiment Station, Radnor, PA. 1996. 7 p.

DUDA, L. L. Seleção genética de árvores de Pinus taeda L. na região de Arapoti, Paraná. 60 f. Dissertação (Mestrado em Engenharia Florestal) - Universidade Federal do Paraná, Curitiba, 2003.

FALKENHAGEN, E. R. Influences of testing sites on the genetic correlations in open-pollinated family trials of Pinus elliottii in South Africa. Theoretical and Applied Genetics. v. 77, n. 6, p. 873 - 880. 1989.

HODGE, G. R.; FERREIRA, A. R.; VEIGA, W. A. Age-age correlations in Pinus taeda in Brazil. In: $\mathbf{2 7}^{\text {th }}$ Southern Forest Tree Improvement Conference at Oklahoma State University in Stillwater, p. 83 - 84, 2003.

KLABIN. Plano de manejo florestal: resumo público Santa Catarina. Otacílio Costa: Klabin, 2009. 12 p.

Plano de manejo florestal 2011: resumo público Telêmaco Borba - PR. Telêmaco Borba: Klabin, 2011.24 p.

NUNES, G. H. S.; REZENDE, G. D. S. P.; RAMALHO, M. A. P.; SANTOS, J. B. Implicações da interação genótipos x ambientes na seleção de clones de eucalipto. Revista Cerne, Lavras, v. 8, n. 1, p. $49-58,2002$.

NUNES, G. H. S. Interação genótipos x ambientes em eucalipto: implicações sobre a seleção e formas de atenuar seu efeito. 160 f. Tese (Doutorado em Genética e Melhoramento de Plantas) - Universidade Federal de Lavras, Lavras, 2000.

PALUDZYSZYN FILHO, E.; SHIMOYAMA, V. R. S.; MORA, A. L. Seleção precoce para incremento simultâneo do crescimento e da qualidade da madeira em Pinus taeda L. Boletim de Pesquisa Florestal. Colombo. Embrapa Florestas, n. 46, p. 31 - 46, 2003.

PALUDZYSZYN FILHO, E.; FERNANDES, J. S. C.; RESENDE, M. D. V. Avaliação e seleção precoce para crescimento de Pinus taeda. Pesquisa Agropecuária Brasileira, Brasília, v. 37, n. 12, p. 1719 1726, 2002.

PALUDZYSZYN FILHO, E.; MORA, A. L.; MAESTRI, R. Interação de genótipos de Pinus taeda L. com locais no sul-sudeste do Brasil. Revista Cerne, v. 7, n. 1, p. 90 - 100, 2001.

RESENDE, M. D. V. Selegen-Reml/Blup: Sistema Estatístico e Seleção Genética Computadorizada via Modelos Lineares Mistos. Colombo: Embrapa Florestas, 2007. 361 p.

Genética biométrica e estatística no melhoramento de plantas perenes. Brasília: Embrapa Informação Tecnológica; Colombo: Embrapa Florestas, 2002a. 975 p.

. Software Selegen-Reml/Blup. Colombo: Embrapa Florestas, (Embrapa Florestas. Documentos, 77), 2002b. 67 p.

RESENDE, M. D. V.; DUARTE, J. B. Precisão e controle da qualidade em experimentos de avaliação de cultivares. Pesquisa Agropecuária Tropical, Goiânia, v. 37, n. 3, p. 182 - 194, 2007.

SUN, X. The adaptability and stability evaluation on introduced families of Pinus taeda. Journal of Anhui Agricultural University. v. 31, n. 3, p. 363 - 367, 2004.

TAMBARUSSI, E. V.; SEBBENN, A. M.; MORAES, M. L. T.; ZIMBACK, L.; PALOMINO, E. C.; MORI, E. S. Estimative of genetic parameters in progeny test of Pinus caribaea Morelet var. hondurensis Barret \& Golfari by quantitative traits and microsatellite markers. Bragantia, Campinas, v. 69, n. 1, p. 39 $-47,2010$.

VENCOVSKY, R.; BARRIGA, P. Genética biométrica no fitomelhoramento. Ribeirão Preto: Sociedade Brasileira de Genética, 1992. 496 p. 
